\title{
Estudios que cambiaron la práctica de la medicina familiar en los últimos 20 años
}

\author{
Practice-changing studies in the last 20 years of family medicine
}

\section{Comentado de:}

Ebell MH, et al. Ann Fam Med. 2018;16(5):436-439. PMID: $30201640^{1}$

\section{Resumen}

Los POEM (por sus iniciales en inglés, Patient-Oriented Evidence that Matters) son comentarios breves de trabajos científicos enfocados a una pregunta clínica relevante, con desenlaces orientados a los pacientes y que tienen el potencial de cambiar la práctica.

Desde su creación en 1994 en la revista estadounidense Annals of Family Medicine, un grupo de autores revisan mensualmente más de 100 revistas biomédicas publicadas en idioma inglés para identificar la literatura médica relevante para la atención primaria de la salud. Su objetivo es seleccionar, entre la vasta cantidad de evidencias que se publican a diario, aquellas que resultan más relevantes para el ejercicio de la medicina familiar, analizarlas en forma crítica e independiente y presentarlas en forma tal que resulte fácil de leer y accesible para otros médicos.
En 2018, tras cumplirse 20 años de su publicación sistemática a partir de 1998, la revista encomendó a los cuatro autores originales que eligieran los POEM más influyentes. La lista final fue consensuada entre los autores a través de un proceso tipo Delphi.

En este artículo se identifican los 20 POEM que tuvieron el puntaje más alto por haber recomendado un cambio mayor y persistente en la práctica, cada uno de los últimos 20 años. Entre ellos se incluyen POEM que recomendaron una intervención novedosa y efectiva, otros que recomendaron abandonar alguna práctica fútil, y otros que recomendaron abandonar una práctica potencialmente dañina. Esta selección ilustra la amplitud del cambio en la práctica en atención primaria de la salud y la necesidad de que los médicos de familia tengan una aproximación sistemática a la literatura biomédica, como la de los POEM, para estar actualizados; especialmente porque muchos de los artículos importantes no aparecen en la literatura específica de atención primaria.

En la Tabla 1, la Tabla 2 y la Tabla 3 se sintetizan los aspectos más relevantes de los artículos seleccionados, con referencias a los estudios originales y los POEM correspondientes.

Tabla 1. Características más relevantes de los estudios sobre diabetes, dislipemia y enfermedad cardiovascular. Notas: ACCF/AHA: Fundación del Colegio Estadounidense de Cardiología y Asociación Estadounidense del Corazón(American College of Cardiology Foundation/American Heart Association); ADA: Asociación Estadounidense de Diabetes (American Diabetes Association); ALAD: Asociación Latinoamericana de Diabetes; ECCA: ensayo clínico controlado aleatorizado; RS: revisión sistemática; FDA: Administración Federal de Medicamentos de los EE.UU; USPSTF: Fuerza de Tareas de Servicios Preventivos de los EE.UU. (US Preventive Services Task Force)

\begin{tabular}{|c|c|c|c|}
\hline $\begin{array}{l}\text { Diseño del estudio } \\
\text { (año) }\end{array}$ & Población & $\begin{array}{c}\text { Intervención/control } \\
\text { o exposición }\end{array}$ & Conclusiones \\
\hline $\begin{array}{l}\text { ECCA }\left(\mathrm{UKPDS}^{2}\right) \\
1998\end{array}$ & $\begin{array}{l}\text { Pacientes con diabetes } \\
\text { mellitus tipo } 2 \text { y sobrepeso }\end{array}$ & $\begin{array}{l}\text { Tratamiento intensivo con insu- } \\
\text { lina, sulfonilureas o metformi- } \\
\text { na (meta de glucemia en ayu- } \\
\text { nas < } 108 \mathrm{mg} / \mathrm{dL} \text { ), vs trata- } \\
\text { miento convencional (meta < } \\
270 \mathrm{mg} / \mathrm{dL} \text { ). }\end{array}$ & $\begin{array}{l}\text { Este trabajo mostró que la metformina } \\
\text { reducía la mortalidad global y la tasa } \\
\text { de eventos tanto microvasculares como } \\
\text { macrovasculares, superando a las sulfoni- } \\
\text { lureas y a la insulina, que sólo reducían } \\
\text { los eventos microvasculares. Esto comen- } \\
\text { zó a instalar a la metformina como droga } \\
\text { de primera línea en personas con diabe- } \\
\text { tes }^{3} \text {. }\end{array}$ \\
\hline $\begin{array}{l}\mathrm{RS}^{4} \\
1999\end{array}$ & $\begin{array}{l}\text { Pacientes con insuficiencia } \\
\text { cardíaca }\end{array}$ & Betabloqueantes vs. placebo & $\begin{array}{l}\text { En pacientes que toleraron su uso, los } \\
\text { betabloqueantes redujeron la mortalidad } \\
\text { global y la tasa de hospitalizaciones y } \\
\text { mejoraron la fracción de eyección }{ }^{5} \text {. } \\
\text { Actualmente, las guías del ACCF/AHA y } \\
\text { la Sociedad Europea de Cardiología reco- } \\
\text { miendan el uso de metoprolol, bisoprolol o } \\
\text { carvedilol para el tratamiento de la insufi- } \\
\text { ciencia cardíaca } 6,7 \text {. }\end{array}$ \\
\hline
\end{tabular}

Continued on next page 


\begin{tabular}{|c|c|c|c|}
\hline $\begin{array}{l}\text { Diseño del estudio } \\
\text { (año) }\end{array}$ & Población & $\begin{array}{c}\text { Intervención/control } \\
\text { o exposición }\end{array}$ & Conclusiones \\
\hline $\begin{array}{l}\mathrm{ECCA}^{8} \\
2001\end{array}$ & $\begin{array}{l}\text { Pacientes con fibrilación } \\
\text { auricular }\end{array}$ & $\begin{array}{l}\text { Control de ritmo (cardioversión } \\
\text { farmacológica o eléctrica) vs. } \\
\text { control de frecuencia cardíaca } \\
\text { (verapamilo) }\end{array}$ & $\begin{array}{l}\text { No se observaron diferencias en cuan- } \\
\text { to a síntomas (palpitaciones, mareos, dis- } \\
\text { nea) al año de seguimiento. Ambas estra- } \\
\text { tegias se consideran igual de efectivas en } \\
\text { la actualidad, tanto en el control de los sín- } \\
\text { tomas como en la prevención de complica- } \\
\text { ciones, pero se tiende a preferir el control } \\
\text { de ritmo en menores de } 65 \text { años }{ }^{9} \text {. }\end{array}$ \\
\hline $\begin{array}{l}\text { ECCA }^{10} \\
2007\end{array}$ & $\begin{array}{l}\text { Pacientes con diabetes } \\
\text { mellitus tipo } 2 \text { no insulini- } \\
\text { zados }\end{array}$ & $\begin{array}{l}\text { Automonitoreo de glucemia vs. } \\
\text { tratamiento habitual }\end{array}$ & $\begin{array}{l}\text { No se observaron diferencias en valo- } \\
\text { res de hemoglobina glicosilada al año. } \\
\text { Sí se documentaron mayores tasas de } \\
\text { hipoglucemia sintomática (en su mayo- } \\
\text { ría leves) en el grupo de automonito- } \\
\text { reo }{ }^{11} \text {. Actualmente hay inconsistencias en } \\
\text { las recomendaciones de distintas socie- } \\
\text { dades científicas, p. ej. la guía de la } \\
\text { ADA recomienda el uso de esta práctica } \\
\text { en los pacientes que reciben tratamien- } \\
\text { to con insulina }{ }^{12} \text {, la ALAD la recomienda } \\
\text { en los pacientes no insulinizados en diver- } \\
\text { sas situaciones }{ }^{13} \text {, y la AAFP explicita su } \\
\text { recomendación en contra del automonito- } \\
\text { reo en forma rutinaria, salvo durante perio- } \\
\text { dos de titulación de fármacos antidiabéti- } \\
\text { cos o cambios importanes en la dieta y la } \\
\text { actividad física }{ }^{14} \text {. }\end{array}$ \\
\hline $\begin{array}{l}\text { ECCA } \\
\left(\text { ACCORD }^{15}\right) \\
2008\end{array}$ & $\begin{array}{l}\text { Pacientes con diabetes } \\
\text { mellitus tipo } 2\end{array}$ & $\begin{array}{l}\text { Control intensivo de glucemia } \\
\text { (meta de hemoglobina glicosi- } \\
\text { lada }<6 \% \text { ) vs. estándar (7 a } \\
8 \% \text { ) }\end{array}$ & $\begin{array}{l}\text { Luego de } 3,5 \text { años, se suspendió el estu- } \\
\text { dio por documentarse mayor mortalidad } \\
\text { en el grupo del tratamiento intensivo. Tam- } \\
\text { bién se hace referencia al ensayo ADVAN- } \\
\mathrm{CE}^{16} \text {, que con metas de hemoglobina gli- } \\
\text { cosilada }<6,5 \% \text { mostró menor tasa de } \\
\text { eventos microvasculares, pero sin diferen- } \\
\text { cias en la incidencia de eventos macrovas- } \\
\text { culares o en la mortalidad global. } \\
\text { Actualmente se proponen metas de } 7 \% \\
\text { en la mayoría de los pacientes, y de } 8 \% \\
\text { en pacientes más frágiles o con muchas } \\
\text { comorbilidades }{ }^{17} \text {. }\end{array}$ \\
\hline $\begin{array}{l}\text { ECCA } \\
\left(\text { ACCORD }^{18}\right) \\
2010\end{array}$ & $\begin{array}{l}\text { Pacientes de } 40 \text { a } 79 \text { años } \\
\text { con diabetes mellitus tipo } \\
2 \text { y enfermedad coronaria, } \\
\text { o al menos dos factores } \\
\text { de riesgo cardiovasculares } \\
\text { adicionales }\end{array}$ & $\begin{array}{l}\text { Estatinas + fibratos vs. sólo } \\
\text { estatinas }\end{array}$ & 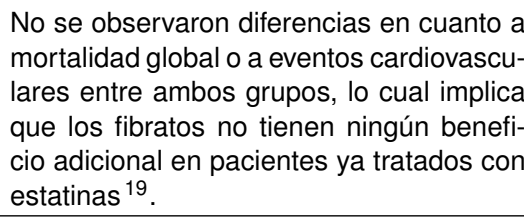 \\
\hline $\begin{array}{l}\mathrm{ECCA}^{20} \\
2011\end{array}$ & $\begin{array}{l}\text { Pacientes con fibrilación } \\
\text { auricular no valvular }\end{array}$ & Rivaroxaban vs. warfarina & $\begin{array}{l}\text { No se observaron diferencias en la inci- } \\
\text { dencia de accidentes cerebrovasculares u } \\
\text { otros eventos tromboembólicos luego de } \\
2 \text { años de seguimiento, ni tampoco en el } \\
\text { riesgo de sangrado. Fue uno de los traba- } \\
\text { jos que empezó a instalar a los llamados } \\
\text { "nuevos anticoagulantes orales. en el trata- } \\
\text { miento de la fibrilación auricular y otras } \\
\text { enfermedades }{ }^{21} \text {. }\end{array}$ \\
\hline
\end{tabular}

Continued on next page 


\begin{tabular}{|c|c|c|c|}
\hline $\begin{array}{l}\text { Diseño del estudio } \\
\text { (año) }\end{array}$ & Población & $\begin{array}{c}\text { Intervención/control } \\
\text { o exposición }\end{array}$ & Conclusiones \\
\hline $\begin{array}{l}\text { Corte transversal } \\
22 \\
2013\end{array}$ & Población general & $\begin{array}{l}\text { Horas de ayuno antes de reali- } \\
\text { zar determinación del perfil lipí- } \\
\text { dico }\end{array}$ & $\begin{array}{l}\text { En este estudio de } 210.000 \text { personas, no } \\
\text { se observaron diferencias en cuanto a los } \\
\text { valores de colesterol total, LDL, HDL y tri- } \\
\text { glicéridos según la cantidad de horas de } \\
\text { ayuno previas, lo cual avala realizar estos } \\
\text { estudios sin ayuno. En caso de resultar } \\
\text { alterados, sobre todo los triglicéridos, se } \\
\text { puede repetir el estudio con una muestra } \\
\text { en ayuno }{ }^{23} \text {. }\end{array}$ \\
\hline $\begin{array}{l}\mathrm{ECCA}^{24} \\
2014\end{array}$ & $\begin{array}{l}\text { Pacientes con enfermedad } \\
\text { cardiovascular establecida } \\
\text { (prevención secundaria) }\end{array}$ & $\begin{array}{l}\text { Estatinas + niacina vs. sólo } \\
\text { estatinas }\end{array}$ & $\begin{array}{l}\text { Similar al ensayo de fibratos, no se obser- } \\
\text { varon diferencias en la mortalidad global } \\
\text { o en el riesgo de desarrollar eventos car- } \\
\text { diovasculares entre ambos grupos. Sí se } \\
\text { observó una disminución del LDL y un } \\
\text { aumento del HDL en el grupo tratado con } \\
\text { niacina, lo cual muestra que no "alcanza" } \\
\text { con mejorar los resultados intermedios o } \\
\text { subrogantes }{ }^{25} \text {. }\end{array}$ \\
\hline $\begin{array}{l}\mathrm{ECCA}^{26} \\
2017\end{array}$ & $\begin{array}{l}\text { Pacientes con diabetes } \\
\text { mellitus y enfermedad } \\
\text { vascular o mayores de } \\
60 \text { años con un factor de } \\
\text { riesgo adicional }\end{array}$ & $\begin{array}{l}\text { Semaglutide (agonista de } \\
\text { GLP-1-[péptido símil gluca- } \\
\text { gón]) vs. placebo }\end{array}$ & $\begin{array}{l}\text { Se observó una disminución de la inciden- } \\
\text { cia de eventos cardiovasculares no letales } \\
\text { (accidentes cerebrovasculares e infartos). } \\
\text { No hubo cambios en mortalidad global. } \\
\text { Este ensayo se hizo luego de una reso- } \\
\text { lución de la FDA en } 2008 \text { que obligaba } \\
\text { a la industria farmacéutica a demostrar la } \\
\text { seguridad y la eficacia de medicamentos } \\
\text { nuevos para la diabetes para prevenir la } \\
\text { enfermedad cardiovascular. } \\
\text { Los análogos de GLP-1 (péptido símil } \\
\text { glucagón) y los antagonistas de SGLT2 } \\
\text { (cotransportador sodio-glucosa), como la } \\
\text { empagliflozina, son los únicos antidiabé- } \\
\text { ticos, más allá de la metformina, que } \\
\text { demostraron reducir la incidencia de even- } \\
\text { tos cardiovasculares en pacientes de alto } \\
\text { riesgo. } \\
\text { Sin embargo la metformina sigue siendo la } \\
\text { droga de primera línea para el tratamiento } \\
\text { de personas con esta enfermedad }{ }^{27} \text {. }\end{array}$ \\
\hline
\end{tabular}

Tabla 2. Características de los estudios más relevantes sobre salud de la mujer

\begin{tabular}{|l|l|l|l|}
\hline $\begin{array}{c}\text { Diseño del estudio } \\
(\mathrm{año})\end{array}$ & Población & $\begin{array}{c}\text { Intervención/control } \\
\text { o exposición }\end{array}$ & \multicolumn{1}{c|}{ Conclusiones } \\
\hline $\begin{array}{l}\text { Cohorte prospecti- } \\
\text { va } 28 \\
2000\end{array}$ & $\begin{array}{l}\text { Mujeres de cualquier } \\
\text { edad }\end{array}$ & $\begin{array}{l}\text { Periodicidad del rastreo de } \\
\text { cáncer de cuello uterino con } \\
\text { papanicolau }\end{array}$ & $\begin{array}{l}\text { Este trabajo mostró que el riesgo de desarro- } \\
\text { Ilar SIL (lesión escamosa intraepitelial) de alto } \\
\text { grado o cáncer en los } 3 \text { años consecutivos } \\
\text { a un papanicolau normal es bajo }(0,66 \% \text { en } \\
\text { mujeres menores de 30 años), lo cual avala la } \\
\text { recomendación actual de realizar papanicolau } \\
\text { cada 3 años } 29,30 .\end{array}$ \\
\hline
\end{tabular}




\begin{tabular}{|c|c|c|c|}
\hline $\begin{array}{c}\text { Diseño del estudio } \\
\text { (año) }\end{array}$ & Población & $\begin{array}{c}\text { Intervención/control } \\
\text { o exposición }\end{array}$ & Conclusiones \\
\hline $\begin{array}{l}\text { ECCA }\left(\mathrm{WHI}^{31}\right) \\
2002\end{array}$ & $\begin{array}{l}\text { Mujeres posmenopáusi- } \\
\text { cas sin histerectomía pre- } \\
\text { via }\end{array}$ & $\begin{array}{l}\text { Terapia de reemplazo hor- } \\
\text { monal (estrógenos equinos } \\
\text { conjugados + progestáge- } \\
\text { nos) o placebo }\end{array}$ & $\begin{array}{l}\text { Luego de } 5 \text { años de seguimiento, las muje- } \\
\text { res asignadas a terapia de reemplazo hormo- } \\
\text { nal tuvieron mayor riesgo de desarrollar enfer- } \\
\text { medad coronaria, cerebrovascular, cáncer de } \\
\text { mama y tromboembolia. Si bien el aumento } \\
\text { del riesgo fue pequeño, esto implica que no es } \\
\text { útil en la prevención primaria de enfermedades } \\
\text { crónicas. Actualmente la recomendación de la } \\
\text { USPSTF es categoría D, en contra de indicar } \\
\text { esta terapia }{ }^{32} \text {. Sin embargo, se sigue usando } \\
\text { para tratar los síntomas de la menopausia en } \\
\text { mujeres sanas, por un tiempo limitado }{ }^{33} \text {. }\end{array}$ \\
\hline $\begin{array}{l}\mathrm{ECCA}^{34} \\
2003\end{array}$ & $\begin{array}{l}\text { Mujeres entre } 30 \text { y } 64 \\
\text { años }\end{array}$ & $\begin{array}{l}\text { Programa de educación en } \\
\text { autoexamen mamario vs. } \\
\text { control habitual }\end{array}$ & $\begin{array}{l}\text { Este ensayo se llevó a cabo en China con alre- } \\
\text { dedor de } 230.000 \text { participantes. No se obser- } \\
\text { varon diferencias en la mortalidad por cáncer } \\
\text { de mama entre ambos grupos, sí una mayor } \\
\text { tasa de detección de lesiones benignas }{ }^{35} \text {. } \\
\text { Actualmente la recomendación de la USPSTF } \\
\text { es categoría D, en contra de realizar esta prác- } \\
\text { tica }^{36} \text {. }\end{array}$ \\
\hline $\begin{array}{l}\text { Cohorte prospecti- } \\
\text { vo }^{37} \\
2004\end{array}$ & $\begin{array}{l}\text { Todas las mujeres sue- } \\
\text { cas y noruegas mayores } \\
\text { de } 30 \text { años }\end{array}$ & $\begin{array}{l}\text { Rastreo de cáncer de } \\
\text { mama con mamografía }\end{array}$ & $\begin{array}{l}\text { Este trabajo mostró un aumento en la inciden- } \\
\text { cia específica de cáncer de mama en mujeres } \\
\text { entre } 50 \text { y } 69 \text { años luego de la introducción del } \\
\text { programa masivo de rastreo con mamografía, } \\
\text { no acompañado de la disminución de la inci- } \\
\text { dencia en mayores de } 69 \text { años que se había } \\
\text { esperado. La tasa de sobrediagnóstico, esti- } \\
\text { mada alrededor de } 33 \% \text {, fue mayor a la de } \\
\text { trabajos previos }^{38} \text {. }\end{array}$ \\
\hline $\begin{array}{l}\mathrm{ECCA}^{39} \\
2005\end{array}$ & Mujeres de 15 a 25 años & 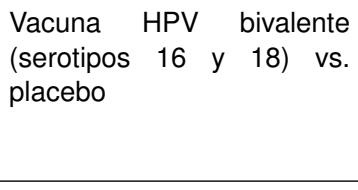 & $\begin{array}{l}\text { Este trabajo mostró la eficacia de la vacuna } \\
\text { para bajar la tasa de infección por HPV } 40 \text {. } \\
\text { Actualmente, se recomienda esta vacuna en } \\
\text { forma rutinaria a los } 11 \text { o } 12 \text { años, y en adul- } \\
\text { tos hasta los } 26 \text { años }{ }^{41} \text {. }\end{array}$ \\
\hline $\mathrm{ECCA}^{42} 2006$ & Mujeres 45 a 69 años & $\begin{array}{l}\text { Rastreo del cáncer de } \\
\text { mama con mamografía } \\
\text { vs. no realizar rastreo con } \\
\text { mamografia }\end{array}$ & $\begin{array}{l}\text { Se observó mayor incidencia de cáncer de } \\
\text { mama en el grupo asignado a mamografía. } \\
\text { Luego de terminado el estudio, se invitó al gru- } \\
\text { po control a hacer mamografías en forma regu- } \\
\text { lar y se realizó un seguimiento adicional por } 15 \\
\text { años más. No se observó una mayor inciden- } \\
\text { cia de cáncer de mama en esas pacientes. Se } \\
\text { estimó que el sobrediagnóstico } \\
\text { fue aproximadamente } 10 \% \text { de los casos }{ }^{43} \text {. }\end{array}$ \\
\hline
\end{tabular}

Tabla 3. Características de los estudios más relevantes sobre cáncer de próstata y otros (apendicitis y neumonía)

\begin{tabular}{|c|c|c|c|}
\hline $\begin{array}{c}\text { Diseño del estudio } \\
(\text { año })\end{array}$ & Población & $\begin{array}{c}\text { Intervención/control } \\
\text { o exposición }\end{array}$ & Conclusiones \\
\hline
\end{tabular}




\begin{tabular}{|c|c|c|c|}
\hline $\begin{array}{c}\text { Diseño del estudio } \\
\text { (año) }\end{array}$ & Población & $\begin{array}{c}\text { Intervención/control } \\
\text { o exposición }\end{array}$ & Conclusiones \\
\hline $\begin{array}{l}\text { ECCA }\left(\mathrm{PLCO}^{44}\right) \\
2009\end{array}$ & Hombres de 55 a 74 años & $\begin{array}{l}\text { Rastreo del cáncer de } \\
\text { prostata con PSA (antí- } \\
\text { geno prostático especí- } \\
\text { fico) anualmente + tacto } \\
\text { rectal vs. control habi- } \\
\text { tual }\end{array}$ & $\begin{array}{l}\text { No se observaron diferencias en la mortalidad } \\
\text { específica por cáncer de próstata entre ambos } \\
\text { grupos. Hubo alta tasa de contaminación ( } 40 \\
\text { a } 52 \% \text { de pacientes del grupo control tuvieron } \\
\text { alguna determinacion de PSA durante el estu- } \\
\text { dio) }{ }^{45} \text {. Actualmente, la recomendación de la } \\
\text { USPSTF es de tipo C (la decisión debe ser indi- } \\
\text { vidual de cada paciente, luego de discutir con } \\
\text { su médico los beneficios y los riesgos de reali- } \\
\text { zar la practica) }{ }^{46} \text {, tomando en cuenta también } \\
\text { otro ensayo que muestra un beneficio pequeño } \\
\text { en la mortalidad } 47 \text {. }\end{array}$ \\
\hline $\begin{array}{l}\mathrm{ECCA}^{48} \\
2016\end{array}$ & $\begin{array}{l}\text { Hombres de } 5 \text { a } 69 \text { años } \\
\text { con cáncer de próstata } \\
\text { localizado }\end{array}$ & $\begin{array}{l}\text { Radioterapia, pros- } \\
\text { tatectomía radical o } \\
\text { vigilancia activa }\end{array}$ & $\begin{array}{l}\text { Luego de } 10 \text { años de seguimiento, no se obser- } \\
\text { varon diferencias en la mortalidad específica por } \\
\text { cáncer de próstata o la mortalidad global, y se } \\
\text { evitaron los efectos adversos del tratamiento, lo } \\
\text { cual avala la vigilancia activa como alternativa. } \\
\text { Sí hubo mayor tasa de enfermedad metastásica } \\
\text { en esos pacientes ( } 3 \% \text { adicional })^{49} \text {. }\end{array}$ \\
\hline $\begin{array}{l}\mathrm{RS}^{50} \\
2012\end{array}$ & $\begin{array}{l}\text { Adultos con apendicitis } \\
\text { aguda }\end{array}$ & $\begin{array}{l}\text { Antibióticos vs. trata- } \\
\text { miento quirúrgico }\end{array}$ & $\begin{array}{l}\text { Se observó una tasa de éxito con el tratamien- } \\
\text { to antibiótico de } 63 \% \text {. Sin embargo, } 30 \% \text { de } \\
\text { los pacientes tuvieron que ser operados al año. } \\
\text { Actualmente se recomienda tratamiento quirúr- } \\
\text { gico para todas las apendicitis }{ }^{51} \text {. }\end{array}$ \\
\hline $\begin{array}{l}\mathrm{RS}^{52} \\
2015\end{array}$ & $\begin{array}{l}\text { Adultos con neumonía hos- } \\
\text { pitalizados }\end{array}$ & Corticoides vs. placebo & $\begin{array}{l}\text { Se observaron mejores resultados con la admi- } \\
\text { nistración de corticoides sistémicos como trata- } \\
\text { miento adyuvante a los antibióticos, entre ellos } \\
\text { menor tasa de mortalidad en aquellos pacientes } \\
\text { con neumonía grave; sin embargo, este tema } \\
\text { sigue siendo controvertido }{ }^{53} \text {. }\end{array}$ \\
\hline
\end{tabular}

\section{Comentario}

La mayoría de los POEM que se resumen en este artículo pueden clasificarse, en líneas generales, dentro de dos categorías. Algunos de ellos implican la recomendación de adoptar una práctica que en su momento fue novedosa y hoy se considera un estándar de tratamiento: por ejemplo, el uso de los betabloqueantes en la insuficiencia cardíaca, o el de la metformina como droga de primera línea en las personas con diabetes. Otros ponen en duda prácticas establecidas, como la terapia de reemplazo hormonal para la prevención de enfermedades crónicas -que fue abandonada luego de la publicación de los resultados del estudio conocido como WHI (por sus iniciales en inglés, Women Health's Initiative)-, el rastreo de cáncer de próstata con antígeno prostático específico, y la periodicidad anual de la mamografía y el PAP para los tamizajes de cáncer mamario y cervical. Estos últimos se encuadran en el concepto de prevención cuaternaria, un componente esencial en la práctica de la medicina familiar para reducir, evitar o paliar los daños de las prácticas médicas ${ }^{54}$ y que despierta inquietantes controversias, algunas de las cuales se encuentran aún en plena discusión a pesar de la evidencia acumulada en el transcurso de las últimas décadas ${ }^{55,56}$.

El articulo resumido nos abre una perspectiva histórica sobre cómo fue cambiando la práctica de la medicina en los últimos años. El movimiento de la medicina basada en la evidencia (MBE) fue en parte responsable de estos cambios, al sostener que las decisiones de la práctica clínica deben basarse en la mejor información disponible, además del criterio profesional del médico y los valores y preferencias de los pacientes.

\section{Conclusiones de los comentadores}

Valoramos los esfuerzos por facilitar la difusión y valoración crítica de conocimientos científicos actualizados que permitan continuar transformando la medicina en una práctica cada vez más humana, transparente y justa, contemplando los derechos y necesidades tanto de los pacientes individuales como de la sociedad en su conjunto. 
Sebastián Sguiglia, Valeria Vietto [ Servicio de Medicina Familiar y Comunitaria, Hospital Italiano de Buenos Aires. sebastian.sguiglia@hospitalitaliano. org.ar, valeria.vietto@hospitalitaliano.org.ar ]

Sguiglia S, Vietto V. Estudios que cambiaron la práctica de la medicina familiar en los últimos 20 años. Evid Actual Pract Ambul. $2020 ; 23(1): e 002006$. Comentado de: Ebell MH, et al. Top 20 POEMs of the Past 20 Years: A Survey of Practice-Changing Research for Family Physicians. Ann Fam Med. 2018;16(5):436-439. PMID: 30201640

\section{Referencias}

1. Ebell MH, Barry HC, Shaughnessy AF, et al. POEMs of the past 20 years: a survey of practice-changing research for family physicians. Ann Fam Med. 2018;16:436-439. Available from: 10.1370/afm.2288.

2. UK Prospective Diabetes Study (UKPDS) Group. Effect of intensive blood-glucose control with metformin on complications in overweight patients with type 2 diabetes (UKPDS 34). Lancet. 1998;352(9131):854-65.

3. Metformin best for obese type-2 diabetes mellitus; 1998. Available from: http://www.essentialevidenceplus.com/content/poem/730.

4. Lechat P, Packer M, Chalon S, et al. Clinical effects of beta-adrenergic blockade in chronic heart failure: a meta-analysis of double-blind, placebocontrolled, randomized trials. Circulation. 1998;98(12):1184-91. Available from: 10.1161/01.cir.98.12.1184.

5. Beta-blockers effective in heart failure; 1999. Available from: http://www.essentialevidenceplus.com/content/poem/1005.

6. Ponikowski P, Voors AA, Anker SD, et al. ESC Guidelines for the diagnosis and treatment of acute and chronic heart failure: The Task Force for the diagnosis and treatment of acute and chronic heart failure of the European Society of Cardiology (ESC). European Journal of Heart Failure. 2016;18(8):891-975.

7. Yancy CW, Jessup M, Bozkurt B, et al. ACCF/AHA guideline for the management of heart failure: executive summary: a report of the American College of Cardiology Foundation/American Heart Association Task Force on practice guidelines. Circulation. 2013;128(16):1810-52.

8. Hohnloser SH, Kuck KH, Lilienthal J. Rhythm or rate control in atrial fibrillation - Pharmacological Intervention in Atrial Fibrillation (PIAF): a randomised trial. Lancet. 2000;356(9244):1789-64. Available from: 10.1016/s0140-6736(00)03230-x.

9. Managing rate and rhythm are similar for atrial fibrillation; 2001. Available from: http://www.essentialevidenceplus.com/content/poem/30261.

10. Farmer A, Wade A, Goyder D, et al. Impact of self monitoring of blood glucose in the management of patients with non-insulin treated diabetes: open parallel group randomised trial. BMJ. 2007;335(7611):132. Available from: 10.1136/bmj.39247.447431.BE.

11. Home glucose monitoring ineffective; 2007. Available from: http://www.essentialevidenceplus.com/content/poem/90953.

12. American Diabetes Association. 5. Facilitating Behavior Change and Well-being to Improve Health Outcomes: Standards of Medical Care in Diabetes-2020. Diabetes Care. 2020;43(Supplement 1):S48-S65. Available from: 10.2337/dc20-S005.

13. Rosas-Guzmán J, Martínez-Sibaja C. Manual de automonitoreo glucémico: Documento de posición de la Asociación Latinoamericana de Diabetes (ALAD). Rev ALAD. 2019;19(Supl 9):103-15. Available from: http://www.revistaalad.com/files/alad_supl_1_19_103-115.pdf.

14. American Academy of Family Physicians. Don't routinely recommend daily home glucose monitoring for patients who have Type 2 diabetes mellitus and are not using insulin.; 2018. Available from: https:/www.choosingwisely.org/clinician-lists/aafp-daily-home-glucose-monitoring-for-patients-withtype-2-diabetes/ [Last access: 02-27-2020].

15. Gerstein HC, Action to Control Cardiovascular Risk in Diabetes (ACCORD) Study Group, et al. Effects of intensive glucose lowering in type 2 diabetes. N Engl J Med. 2008;358(24):2545-59. Available from: 10.1056/NEJMoa0802743.

16. The ADVANCE Collaborative Group. Intensive blood glucose control and vascular outcomes in patients with type-2 diabetes. $\mathrm{N}$ Engl $\mathrm{J}$ Med. 2008;358(24):2560-2572. Available from: 10.1056/NEJMoa0802987.

17. Intensive glucose control in type-2 diabetes mellitus may be harmful; 2008. Available from: http://www.essentialevidenceplus.com/content/poem/ 100825.

18. The ACCORD Study Group. Effects of combination lipid therapy in type 2 diabetes mellitus. N Engl J Med. 2010;362(17):1563-74. Available from: 10.1056/NEJMoa1001282.

19. Statin + fenofibrate no better than statin alone in type-2 diabetes mellitus; 2010. Available from: http://www.essentialevidenceplus.com/content/poem/ 120501.

20. Patel MR, ROCKET AF Investigators, et al. Rivaroxaban versus warfarin in nonvalvular atrial fibrillation. N Engl J Med. $2011 ; 365(10): 883-91$. Available from: 10.1056/NEJMoa1009638.

21. Rivaroxaban similar to warfarin in patients with NVAF; 2011. Available from: http://www.essentialevidenceplus.com/content/poem/131005.

22. Sidhu D, Naugler C. Fasting time and lipid levels in a community-based population. A cross-sectional study. Arch Intern Med. $2012 ; 172(22): 1707-10$. Available from: 10.1001/archinternmed.2012.3708.

23. Fasting not necessary before lipid panels; 2013. Available from: http://www.essentialevidenceplus.com/content/poem/150134.

24. The HPS2-THRIVE Collaborative Group. Effects of extended-release niacin with laropiprant in high-risk patients. N Engl J Med. 2014;371(3):203212. Available from: $10.1056 /$ NEJMoa1300955.

25. Niacin doesn't improve outcomes in patients with vascular disease; 2014. Available from: http://www.essentialevidenceplus.com/content/poem/ 160901.

26. Marso SP, SUSTAIN-6 Investigators, et al. Semaglutide and cardiovascular outcomes in patients with type 2 diabetes. $\mathrm{N}$ Engl $\mathrm{J}$ Med. 2017;376(9):1834-44. Available from: 10.1056/NEJMc1615712.

27. Semaglutide reduces CV events in high-risk patients with type-2 diabetes mellitus; 2017. Available from: http://www.essentialevidenceplus.com/ content/poem/190106.

28. Sawaya GF, Kerlikowske K, lee NC, et al. Frequency of cervical smear abnormalities within 3 years of normal cytology. Obstet Gynecol. 2000;96(2):219-23. Available from: 10.1016/s0029-7844(00)00882-6.

29. 3 years between PAP smears adequate for many women; 2000. Available from: http://www.essentialevidenceplus.com/content/poem/21184.

30. US Preventive Services Task Force. Final Recommendation Statement: Cervical Cancer: Screening. U.S. Preventive Services Task Force. JAMA. 2018;320(7):674-686. Available from: 10.1001/jama.2018.10897.

31. Writing Group for the Women's Health Initiative Investigators. Risks and benefits of estrogen plus progestin in healthy postmenopausal women. Principal results from the Women's Health Initiative randomized controlled trial. JAMA. 2002;288(3):321-33. Available from: 10.1001/jama.288.3.321.

32. U S Preventive Services Task Force. Final Recommendation Statement: Hormone Therapy in Postmenopausal Women: Primary Prevention of Chronic Conditions. JAMA. 2017;318(22):2224-2233. Available from: 10.1001/jama.2017.18261.

33. Hormone replacement overall not beneficial; 2002. Available from: http://www.essentialevidenceplus.com/content/poem/40926.

34. Thomas DB, Gao DL, Ray RM, et al. Randomized trial of breast self-examination in Shanghai: final results. J Natl Cancer Inst. $2002 ; 94(19): 1445-57$. Available from: 10.1093/jnci/94.19.1445.

35. Self breast exam doesn't reduce mortality; 2003. Available from: http://www.essentialevidenceplus.com/content/poem/50166.

36. US Preventive Services Task Force. Screening for Breast Cancer: U.S. Preventive Services Task Force Recommendation Statement. Ann Intern Med. 2009;151(10):716-26. Disponible en. Available from: 10.7326/0003-4819-151-10-200911170-00008. 
37. Zahl PH, Strand BH, Maehlen J. Incidence of breast cancer in Norway and Sweden during introduction of nationwide screening: prospective cohort study. 2004;328(7445):921-24. Available from: 10.1136/bmj.38044.666157.63.

38. Breast cancer screening results in overdiagnosis; 2003. Available from: http://www.essentialevidenceplus.com/content/poem/60764.

39. Harper DM, Franco EL, Wheeler C. Efficacy of a bivalent L1 virus-like particle vaccine in prevention of infection with human papillomavirus types 16 and 18 in young women: a randomised controlled trial. Lancet. 2004;364(9447):1757-65. Available from: 10.1016/S0140-6736(04)17398-4.

40. Vaccine prevents HPV infection; 2004. Available from: http://www.essentialevidenceplus.com/content/poem/70144.

41. Meites E, Szilagyi PG, Chesson HW, et al. Human Papillomavirus Vaccination for Adults: Updated Recommendations of the Advisory Committee on Immunization Practices. MMWR Morb Mortal Wkly Rep. 2019;68:698-702. Available from: 10.15585/mmwr.mm6832a3.

42. S SZ, Andersson I, Janzon L. Rate of over-diagnosis of breast cancer 15 years after end of Malmo mammographic screening trial: follow-up study. 2006;332(7543):689-92. Available from: 0.1136/bmj.38764.572569.7C.

43. Mammography results in overdiagnosis; 2006. Available from: http://www.essentialevidenceplus.com/content/poem/80654

44. Andriole GL, Crawford ED, Grubb RL, PLCO Project Team. Mortality results from a randomized prostate-cancer screening trial. N Engl J Med. 2009;360(13):1310-1319. Available from: 10.1056/NEJMoa0810696.

45. PSA screening does not reduce mortality from prostate cancer; 2009. Available from: http://www.essentialevidenceplus.com/content/poem/110501.

46. US Preventive Services Task Force. Screening for Prostate Cancer: US Preventive Services Task Force Recommendation Statement. JAMA. 2018;319(18):1901-1913. Disponible en. Available from: 10.1001/jama.2018.3710.

47. Schröder FH, ERSPC Investigators. Screening and prostate cancer mortality: results of the European Randomised Study of Screening for Prostate Cancer (ERSPC) at 13 years of follow-up. Lancet;9959(2027-2035).

48. Hamdy FC, Donovan JL, Lane JA, et al. 10-Year outcomes after monitoring, surgery, or radiotherapy for localized prostate cancer. N Engl J Med. 2016;375(15):1415-1424. Available from: 10.1056/NEJMoa1606220.

49. Active surveillance for localized prostate cancer: no increased mortality, but higher rates of clinical progression; 2016. Available from: http://www. essentialevidenceplus.com/content/poem/181203.

50. Varadhan KK, Neal KR, Lobo DN. Safety and efficacy of antibiotics compared with appendicectomy for treatment of uncomplicated acute appendicitis. BMJ. 2012;344:e2156. Available from: 10.1136/bmj.e2156.

51. Therapy with antibiotics is an option for early uncomplicated appendicitis; 2012. Available from: http://www.essentialevidenceplus.com/content/poem/ 140653.

52. Siemieniuk R, Meade MO, Alonso-Coello P, et al. Corticosteroid therapy for patients hospitalized with community-acquired pneumonia. Ann Intern Med. 2015;163(7):519-28. Available from: 10.7326/M15-0715.

53. Steroids beneficial as adjunctive treatment for community-adquired pneumonia; 2015. Available from: http://www.essentialevidenceplus.com/content/ poem/171210.

54. Kopitowski K. Prevención cuaternaria: se pueden y se deben limitar los daños por la actividad sanitaria. Rev Hosp Ital B Aires. 2013;33(3):90-95. Available from: https://www.hospitalitaliano.org.ar/multimedia/archivos/servicios attachs/8937.pdf.

55. Kopitowski K. La paradoja del octubre rosa. Evid Actual Pract Ambul. 2019;22(3):e002018. Available from: http://www.evidencia.org.ar/index.php/ Evidencia/article/view/4241

56. Malanda U, SD SB, Nijpels G. Self-monitoring of blood glucose in noninsulin-using type 2 diabetic patients: it is time to face the evidence. Diabetes Care. 2013;36(1):176-8. Available from: 10.2337/dc12-0831. 